\title{
EXPOSURE RELATED MORTALITY FROM OBSTRUCTIVE LUNG DISEASES AMONG WORKERS IN THE NORWEGIAN SILICON CARBIDE INDUSTRY
}

Merete Drevvatne Bugge, ${ }^{1}$ Solveig Føreland, ${ }^{2}$ Kristina Kjærheim, ${ }^{3}$ Wijnand Eduard, Jan Ivar Martinsen, ${ }^{3}$ Helge Kjuus ${ }^{1}{ }^{1} \mathrm{NIOH}$, Oslo, Norway; ${ }^{2}$ Norwegian University of Science and Technology, Trondheim, Norway; ${ }^{3}$ Cancer Registry of Norway, Oslo, Norway

10.1136/oemed-2011-100382.210

Objectives An excess mortality from asthma, chronic bronchitis, and emphysema, is previously reported from the silicon carbide $(\mathrm{SiC})$ industry. Our objective was to study mortality from obstructive lung diseases (OLD) with relation to specific exposure factors, using a revised and extended job exposure matrix (JEM). Methods Cumulative exposures to quartz, cristobalite, $\mathrm{SiC}$ particles, and $\mathrm{SiC}$ fibres were assigned to a cohort of 1687 long-term $\mathrm{SiC}$ industry workers, using a detailed JEM. Standardised mortality ratios (SMRs) for underlying causes of death, 1951-2007, were calculated, and Poisson regression analyses of OLD as underlying or contributing cause of death were performed using categorised and log-transformed cumulative exposure variables. Results The SMR of OLD was increased at the highest level of cumulative exposure to all investigated exposure factors. In the internal analyses of OLD as underlying or contributing cause of death, a twofold increased risk was observed at both medium and high level of cumulative exposure to $\mathrm{SiC}$ particles, with a significant trend. In a model with log-transformed cumulative exposure variables, $\mathrm{SiC}$ particles was the exposure factor with the most stable increased risk estimate when controlled for other exposure factors, among workers with less than 15 years of total employment duration. Cristobalite seemed to be the most important exposure factor among workers with more than 15 years total employment.

Conclusions Exposure to $\mathrm{SiC}$ and crystalline silica may contribute to OLD development among $\mathrm{SiC}$ industry workers in different time windows, and possibly through different mechanisms. 